2 A comparison of pain registration by Visual Analog Scale and Numeric

3 Rating Scale - a cross-sectional study of primary triage registration

4

5 Jofrid Kollltveit ${ }^{1 *}$, Malin Osaland ${ }^{1 *}$, Marianne Reimers ${ }^{2}$, Magnus Berle ${ }^{3}$

8 1. University of Bergen - Faculty of Medicine, Bergen, Norway

9 2. Haukeland University Hospital, Emergency Clinic

10 3. Haukeland University Hospital, Department of Gastrointestinal Surgery and

11 University of Bergen, Department of Clinical Medicine.

$12 *$ The authors have contributed equally

14 Correspondance to: Magnus.berle@uib.no

\title{
17 Background
}

18 Pain is a subjective sensation; self-reporting is important for quantifying pain

19 intensity. There are several different validated tools for this, such as Visual Analog

20 Scale and Numeric Rating Scale. In the clinic, these terms are often used as

21 equivalent. The objective of this study was to examine correlation and agreement

22 between the pain registration tools in triage in an emergency department.

\section{Materials and Methods}

25 The study was performed in the Department of Emergency Medicine at Haukeland

26 University Hospital in the period June-August 2019. We registered the pain score

27 with two tools in 200 unselected patients in emergency admission with pain. In

28 addition, we registrered gender, age, triage and general department affiliation. 


\section{Results}

31 We found a strong correlation between the pain registration tools by Spearmans

32 correlation test $(\mathrm{rho}=0,930, \mathrm{p}<0,001)$. There were no significant difference

33 between the pain registration tools within the subgroups. Bland-Altman analysis

34 show agreement between the two pain registration tools.

\section{Conclusions}

37 In an Emergency Department triage is it acceptable to use Visual Analog Scale and

38 Numeric Rating Scale as equivalent, as long as the correct terminology is used.

40 Keywords: Triage, Pain, Pain score, VAS, NRS,

\section{Background}

43 Pain is a subjective sensation, which is important to quantify in a clinical setting (1,

44 2). Pain score is a part of defining the degree of urgency of diagnostics and

45 treatment, and is important for evaluating the effect of treatment. There are several

46 validated tools to rate pain, as Visual Analog Scale (VAS) and Numeric Rating

47 Scale (NRS) $(2,3)$. Using VAS, the patient defines the degree of pain on a visual

48 scale without numeric values, while the values are visible on the back side for the

49 registrar. NRS is a verbal numeric scale, where the patient grades their own pain on

50 a scale between 0 and 10 . Both methods are much used in clinical practice and

51 research (3). In the clinic, the terms are often used as interchangeable, and NRS is

52 in general noted as VAS (4). Several studies have shown a strong statistical

53 correlation between VAS and NRS scores $(1,5,6,7)$. Some studies show good

54 agreement between methods $(1,6)$ while others find a larger discrepancy $(5,7,8)$.

55 The objective of this study was to examine correlation and agreement between 
56 VAS and NRS score in patients admitted with pain to an emergency department in

57 Norway.

\section{Materials and Methods}

60 We performed a cross-sectional study of pain in primary triage registration at the

61 emergency department at Haukeland University Hospital in the period June to

62 August 2019. As a part of the primary triage at admission, the nurse collected pain

63 score by use of NRS. The nurse asked the patient to grade their pain with an integer

64 between 0 and 10 , where 0 is no pain and 10 is the worst thinkable pain. After the

65 nurse had finished the triage, the patients were asked to participate in a study. After

66 oral approval, the patient was again scored with VAS. We used a horizontal VAS-

67 ruler (0-100 mm) with the endpoints "no pain" to the left and "worst thinkable

68 pain" to the right. The patient graded their pain with a moveable plastic marker to

69 their pain level. We transcribed the value in mm and converted to the closest

70 integer (0-10). The following variables were registered on paper: VAS, NRS,

71 gender, age, triage and general department affiliation.

72 The target population were patients with pain in triage in the Emergency

73 Department. We made a general estimation in advance on the number of patients to

74 include, based on presumed sufficient material and a general achivable number.

75200 unselected patients with pain were included, no clinical groups of patients

76 were excluded. Exclusion criteria were age under 18 years, lack of competence to

77 consent, cognitive failure as well as lack of lingual or motor skills.

78 The study included patients after informed oral consent. We have in our study

79 design refrained from written informed consent to reduce the amount of registered

80 personal information. The patient information was anonymized immediately after

81 inclusion. The procedure of inclusion was made in agreement with the Data

82 Protection Officer of the Hospital and the Regional Ethics Board of Western

83 Norway. 
84 We predefined a null hypothesis of no significant difference between VAS and

85 NRS value. A priori acceptable clinical difference between the scores were defined

86 to a maximum of $+/-1$, based on repeatability of test-retest of VAS (1). The

87 collected data were organized in MS Excel, then analyzed in SPSS v2.5. We

88 utilized Spearman correlation coefficient and Bland-Altman plot to study

89 correlation and agreement between VAS and NRS value. Kruskal-Wallis test was

90 used for the subgroup analyses between VAS and NRS for gender (female/male),

91 age (over/under 30 years old), triage (green/yellow/orange) and general department

92 affiliation (surgery/medicine)

\section{Results}

95 Table 1 shows descriptive statistics with mean for VAS and NRS with standard

96 deviation, as well as values for statistical tools. Spearman correlation coefficient

$97($ rho $=0,93)$ show strong correlation between VAS and NRS value $(\mathrm{p}<0,001)$.

98 Subgroup analysis by Kruskal-Wallis test does not show significant differences

99 between VAS and NRS-value within the subgroups ( $\mathrm{p}<0,05)$. Bland-Altman plot

100 (Figure 1) show agreement between VAS and NRS-value as pain scores.

\section{Discussion}

103 The strength of this study is that pain was reported by VAS short time after NRS

104 by standardized questions in such a way that the information to the patient group

105 was the same. The data collection was in daytime and evening in an un-selected

106 patient group to ensure a more representative material.

107 The study has some limitations. Presenting VAS short time after NRS may

108 introduce a bias. Collection of oral informed consent creates an emphasis on the

109 fact that this is a study, with risk of patients attempting to answer the same. All

110 patients were presented to NRS before VAS, where the triage nurse collected NRS-

111 value while the VAS-value was collected by two medical students. We do only 
112 present a single time point and the data cannot tell of changes in VAS versus NRS

113 over time. We have not examined which method is the better, only whether the two

114 methods produce equal results. Earlier studies establish a consensus that NRS is

115 easier to perform than VAS $(2,9)$. The results of this study do not establish that the

116 method is equal in all populations. There are similar studies in other populations,

117 among them orthopedic patients and cancer patients. These studies show good

118 correlation between VAS and NRS $(10,11)$.

119 There are conflicting studies on the agreement between NRS and VAS $(1,5,6,7$,

120 8). Our results support the studies showing good correlation and agreement

121 between the methods $(1,6)$. We cannot abandon the null hypothesis of no

122 significant difference and the limits of agreement in Bland-Altman plot is whitin

123 the a priori clinical acceptable difference. This can indicate that the methods are

124 interchangeable. NRS being noted as VAS will not cause errors in the clinic but we

125 would advocate the use of proper terminology.

\section{Conclusions}

128 The study shows a strong statistical correlation and a good agreement for reported

129 scores between VAS and NRS. In an Emergency Department triage is it acceptable

130 to use Visual Analog Scale and Numeric Rating Scale as equivalent, as long as the

131 correct terminology is used.

133 List of abbreviations

134 VAS - Visual Analog Scale

135 NRS - Numeric Reported Scale

\section{Declarations}

\section{Ethics approval and consent to participate}


139 The study was approved by the Data Protection Officer of the Hospital and the

140 Regional Ethics Board of Western Norway, approval REK Vest (2019/484).

\section{Consent for publication}

142 Patients were included by oral consent to limit registered personal information in

143 accordance with the Data Protection Officer and the Regional Ethics Committee.

\section{Availability of Data}

145 The data in anonymized form is included as supplementary data.

\section{Competing interests}

147 The authors declare that they have no competing interests.

\section{Funding}

149 The article costs is funded by University of Bergen grants to MB.

\section{Authors' contributions}

151 Patient inclusion and data collection JK, MO. Project plan, implementation and

152 data analysis JK, MO, MR, MB. Writing of manuscript JK, MO, MB. Original idea

153 MB. All Authors read and approved the final manuscript.

\section{Figure and table text}

157 Table 1: Descriptive statistics and statistical calcaltions

158 Figure 1: Bland-Altman plot of NRS vs VAS.

159 Additional file 1: Anonymized registered data

\section{References}


1. Bahreini M, Jalili M, Moradi-Lakeh M. A comparison of three self-report pain scales in adults with acute pain. J Emerg Med 2015; 48: 10-8. scales in adults: Which to use? Am J Emerg Med 2018; 36: 707-14

3. Ferreria-Valenta MA, Pais-Ribeiro JL, Jensen MP. Validity of four pain intensity rating scales. Pain 2011; 152: 2399-404

4. Faiz KW. VAS - visuell analog skala. Tidsskr Nor Legeforen 2014; 134: 323 scale with the visual analogue scale for the measurement of acute pain. Emergency Medicine 2003; 15: 441-6.

6. Bijur PE, Latimer CT, Gallagher EJ. Validation of a Verbally Administered Numerical Rating Scale of Acute Pain for Use in the Emergency Department. Acad Emerg Med 2003; 10: 390-2

7. Mohan H, Ryan J, Whelan B et al. The end of the line? The Visual Analogue Scale and Verbal Numerical Rating Scale as pain assessment tools in the emergency department. Emerg Med J 2010; 27: 372-5 Pain Measurement: A Ratio Measure? Pain Pract 2003; 3: 310-6

9. Safikhani S, Gries KS, Trudeau JJ et al. Response scale selection in adult pain measures: results from a literature review. J Patient Rep Outcomes 2018; $2: 40$

10. Paice JA, Cohen FL. Validity of a verbally administered numeric rating scale to measure cancer pain intensity. Cancer Nurs 1997; 20: 88-93

11. Alghadir AH, Anwer S, Iqbal A et al. Test-retest reliability, validity, and minimum detectable change of visual analog, numerical rating, and verbal rating scales for measurement of osteoarthritic knee pain. J Pain Res 2018; 11: $851-6$ 
medRxiv preprint doi: https://doi.org/10.1101/2020.11.03.20225367; this version posted November 4, 2020. The copyright holder for this preprint (which was not certified by peer review) is the author/funder, who has granted medRxiv a license to display the preprint in perpetuity.

It is made available under a CC-BY 4.0 International license.

193

194

195

196

197 
$3 \mid$ medRxiv preprint doi: https://doi.org/10.1101/2020.11.03.20225367; this version posted November 4, 2020. The copyright holder for this preprint (which was not certified by peer review) is the author/funder, who has granted medRxiv a license to display the preprint in perpetuity.

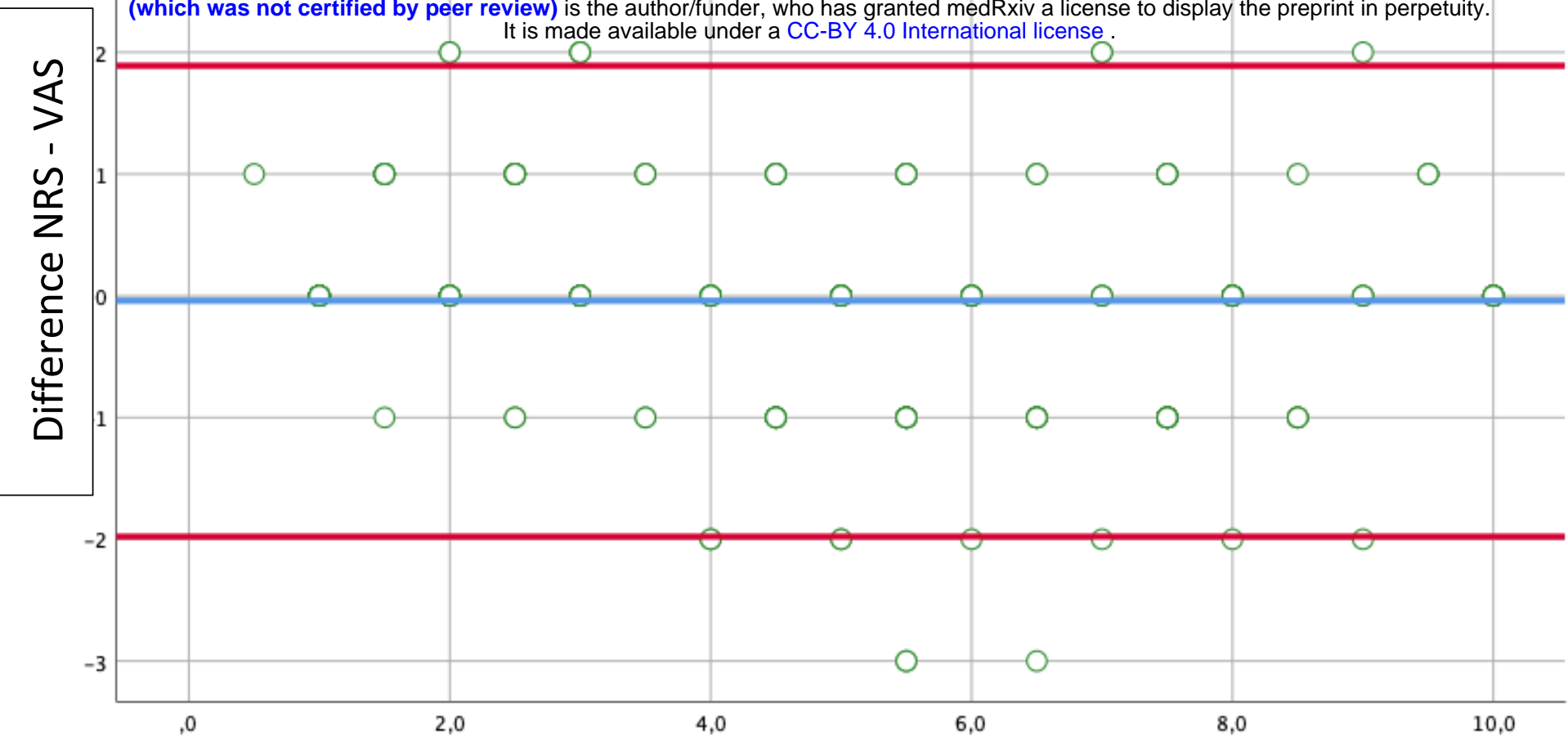

Average VAS NRS 